\title{
Spin Foam Models of Matter Coupled to Gravity
}

\author{
A. Miković 用 \\ Departamento de Matemática e Ciências de Computação, Universidade \\ Lusofona, Av. do Campo Grande 376, 1749-024 Lisboa, Portugal
}

\begin{abstract}
We construct a class of spin foam models describing matter coupled to gravity, such that the gravitational sector is described by the unitary irreducible representations of the appropriate symmetry group, while the matter sector is described by the finite-dimensional irreducible representations of that group. The corresponding spin foam amplitudes in the four-dimensional gravity case are expressed in terms of the spin network amplitudes for pentagrams with additional external and internal matter edges. We also give a quantum field theory formulation of the model, where the matter degrees of freedom are described by spin network fields carrying the indices from the appropriate group representation. In the non-topological Lorentzian gravity case, we argue that the matter representations should be appropriate $S O(3)$ or $S O(2)$ representations contained in a given Lorentz matter representation, depending on whether one wants to describe a massive or a massless matter field. The corresponding spin network amplitudes are given as multiple integrals of propagators which are matrix spherical functions.
\end{abstract}

*E-mail address: amikovic@ulusofona.pt. An associate of Institute of Physics, P.O.Box 57, 11001 Belgrade, Yugoslavia 


\section{Introduction}

The Barrett-Crane spin foam state sum models of four-dimensional quantum gravity [1], 2, 3, [4], represent a promising approach for constructing a quantum theory of gravity. These are non-topological state sum models, and they give discrete spacetime three-geometry to three-geometry transition amplitudes [5]. One can consider these models as examples of regularized path integral approach to quantum gravity. The spacetime is described by a simplical complex, and the state sum is over colored triangulations. This approach is a category theory generalization of the Regge calculus, where the basic category involved is the category of representations of the appropriate symmetry group. This group is taken to be $S O(d)$ in the $d$-dimensional Euclidian gravity case, or $S O(d-1,1)$ in the Lorentzian case. Other groups, like anti de-Sitter, can be considered as well [5].

One can also think of the spin foam models as the higher-dimensional generalizations of the string theory matrix models, since the spin foam amplitudes are given by a field theory Feynman diagrams which are dual to spacetime triangulations $[8,0,10,11,50$. In the $d=2$ case one recovers the string theory matrix models [5, [12].

The standard way of making the spin foam partition function and the amplitudes finite is by passing to the corresponding quantum group category [6, 6, 2]. However, it was discovered in [3, 团 that even in the Lie group case, the $\mathrm{BC}$ model could be made finite by changing the field theory propagator. It has been proven that the BC Euclidian model is perturbatively finite [13, and recently this has been proven for the BC Lorentzian model [14], i.e. the amplitude for any non-degenerate finite triangulation of the spacetime is finite.

In order to make a spin foam model a serious candidate for a quantum theory of gravity, like string theory, one should be able to incorporate matter. Mathematically this would mean enlarging the category of representations, by adding a new set of representations corresponding to the matter fields. The first proposal of this kind was made by Crane [15], who suggested a tensor category of unitary representations for the quantum Lorentz group. In this proposal a subset of the irreducible representations (irreps) corresponds to the gravitational degrees of freedom (DOF), while the rest corresponds to the matter DOF. The corresponding state sum model is topological, which is justified as an acceptable feature of the theory at high energies. Then one conjectures that the local dynamics would appear at low energies, by some 
sort of symmetry breaking mechanism. In a more recent paper [16, Crane has proposed to interpret the graphs of the field theory formulation which do not correspond to triangulations of manifolds, as triangulations of manifolds with conical singularities, and then to interpret these singularities as matter DOF. These are interesting and very geometrical proposals; however, it is not clear how to relate these matter DOF to the particle field theory description in terms of fields carrying pseudo-unitary finite-dimensional Lorentz irreps.

In this paper we give a formulation of a spin foam model with matter inspired by the particle field theory formalism [17]. We consider a class of spin foam models describing matter coupled to gravity, where the gravitational sector is described by the unitary irreps of the appropriate symmetry group, while the matter sector is described by the finite dimensional irreps of that group. In the non-topological four-dimensional Lorentzian gravity case, the gravitational sector will be described by the $\mathrm{BC}$ model, while in the matter sector we will argue that one needs to take appropriate $S O(3)$ or $S O(2)$ representations contained in a given finite-dimensional Lorentz irrep. The construction we present here naturally follows from the field theory formulation of the spin foam amplitudes [5]. It was shown there that a spin foam transition amplitude can be represented as an matrix element of an evolution operator, where the initial and the final states correspond to the three-dimensional spin networks (or spin nets for short) induced by the boundary triangulations. One can represent these spin net states as the spin net operators acting on an appropriate Fock space vacuum. One can think of these spin net operators as the second quantized spin net wave-functions from the canonical loop gravity [18]. Since the spin net wave-functions carrying fermionic matter have been introduced in the context of canonical loop gravity [21, 22], it is not difficult to generalize these results to the second quantization formalism [, so that the matter insertions at the spin net vertices become appropriate creation/annihilation operators.

In section 2 we give basic definitions and formulas concerning the spin net functions. In section 3 we give basics on spin foams, and explain how the spin foam amplitudes are related to the spin net amplitudes. In section

\footnotetext{
${ }^{\dagger}$ The spin network quantum field theory is an example of what is traditionally called a third quantization of gravity, because it allows for more then one universe (a boundary triangulation) in the formalism [5]. Since general relativity is a classical field theory, then the expression "second quantization" is a more appropriate terminology. However, the exact relationship between the loop gravity formulation and the spin-foam formulation is not yet fully understood.
} 
4 we construct the fermionic spin net operators and states, and describe the general structure of the interaction with the gravitational background. In section 5 we discuss the fermions in the Euclidian gravity case. We show that in the non-topological gravity case, the corresponding spin-net amplitudes can be written as the multiple integrals of propagators over the homogenous space $S^{3}=S O(4) / S O(3)$, where the fermionic propagator is given by a matrix zonal spherical function. These results are straightforwardly generalized to the Lorentzian gravity case in the next section. In section 7 we discuss the general structure of the interaction terms for the case of matter fields of arbitrary spins. In the non-topological gravity case, the spin-net matter propagators are described by the corresponding matrix spherical functions. We also argue that the spin-net propagators for the analogues of the massless gauge bosons are the matrix spherical functions restricted to an $S O(2)$ subgroup. In section 8 we present our conclusions.

\section{Spin net functions and amplitudes}

We review the results on spin nets 23] from a point of view which is most suitable for the type of generalizations we want to make.

Consider square-integrable functions $\Psi$ on $G^{E}$, where $G$ is a Lie group and $E$ is a natural number. A gauge invariant function is determined by an oriented graph $\Gamma$ with $V$ vertices and $E$ edges, where a group element is associated to each edge $e \in E$. If we label the vertices of $\Gamma$ as $v_{j}, 1 \leq j \leq V$, then the edges connecting the vertices $j$ and $k$ can be denoted as $e_{j k}$, and the corresponding group elements as $g_{j k}$. Orientation is such that $g_{j k}=g_{k j}^{-1}$. A function $\Psi_{\Gamma}$ associated to the graph $\Gamma$ is gauge invariant if

$$
\Psi_{\Gamma}\left(g_{j k}\right)=\Psi_{\Gamma}\left(h_{j} g_{j k} h_{k}^{-1}\right)
$$

for all $h_{j}, h_{k} \in G$.

By using the Peter-Weyl theorem, one can show that a gauge invariant function $\Psi_{\Gamma}$ can be expanded as

$$
\Psi_{\Gamma}\left(g_{1}, \ldots, g_{E}\right)=\sum_{\Lambda, i} c_{\Lambda, i} \Phi_{\Gamma, \Lambda, i}\left(g_{1}, \ldots, g_{E}\right)
$$

where $\Lambda=\left\{\Lambda_{1}, \ldots, \Lambda_{E}\right\}$ denote the unitary irreps of $G$ labeling the edges of $\Gamma$, and $i=\left\{i_{1}, \ldots, i_{V}\right\}$ denote the intertwiners associated to the vertices of $\Gamma$. 
The basis functions $\Phi$ are called the spin net functions, and they are given by

$$
\prod_{e \in E} D^{(\Lambda)^{\beta_{e}}}{ }_{\alpha_{e}}\left(g_{e}\right) \prod_{v \in V} C_{\ldots}^{\ldots\left(i_{v}\right)}
$$

where $D^{(\Lambda)}(g)$ is the representation matrix of $g$, acting in the space $V(\Lambda)$. All the representation indices $\alpha_{e}$ and $\beta_{e}$ are contracted by the indices from the product of the intertwiner components. Contraction is defined as $X^{\alpha} Y_{\alpha}=$ $C^{\alpha \beta} X_{\alpha} Y_{\beta}$, where $C^{\alpha \beta}$ are the components of the $V(\Lambda) \rightarrow V\left(\Lambda^{*}\right)=V^{*}(\Lambda)$ intertwiner, and $V^{*}$ is the dual vector space. Since any intertwiner can be represented as a linear map $V\left(\Lambda_{1}\right) \otimes \cdots \otimes V\left(\Lambda_{n}\right) \rightarrow \mathbf{C}$, we denote the corresponding vector space as $\operatorname{Inv}\left(\Lambda_{1}, \ldots, \Lambda_{n}\right)$.

Given a spin net function $\Phi_{\Gamma, \Lambda, i}\left(g_{e}\right)$, one can obtain a group invariant associated to the spin net

$$
A(\Gamma, \Lambda, i)=\Phi_{\Gamma, \Lambda, i}\left(g_{1}=g_{0}, \ldots, g_{E}=g_{0}\right) \quad,
$$

where $g_{0}$ is the identity element of $G$. We will call the number $A$ the amplitude for the spin net $(\Gamma, \Lambda, i)$. These objects are the basic building blocks of the state sum amplitudes. Since $D_{\alpha}^{\beta}\left(g_{0}\right)=\delta_{\alpha}^{\beta}$, the amplitude $A$ is simply the product of the intertwiners $C_{\ldots} \ldots\left(i_{v}\right)$ for all vertices $v \in V$, with all the representation indices being contracted. The graphs $\Gamma$ which are relevant for the state sum models are the one skeletons of $d$-simplices, as well as the $\Theta_{d}$ graphs (two points joined by $d$ edges).

In the case of open spin nets, the above results can be easily generalized. The amplitude $A$ for an open spin net will be a group tensor given by the product of intertwiners for the vertices, where all the representations indices are contracted, except the indices corresponding to the irreps labelling the external edges. This can be represented as

$$
A_{\alpha_{1} \ldots}^{\beta_{2} \ldots}(\Gamma, \Lambda, i)=C_{\cdots}^{\ldots\left(i_{1}\right)} \ldots C_{\cdots \ldots \alpha_{1} \ldots}^{\ldots\left(i_{k}\right)} \cdots C_{\cdots \ldots \beta_{2} \ldots\left(i_{l}\right)} \ldots C_{\cdots}^{\ldots\left(i_{V}\right)} .
$$

Hence the amplitude for an open spin net is not an invariant, but it is a tensorial quantity. One can construct a group invariant by contracting the external edge indices with the vectors and the co-vectors from the external irrep vector spaces

$$
S(\Gamma, \Lambda, i, v, u)=A_{\alpha_{1} \ldots}^{\beta_{2} \ldots}(\Gamma, \Lambda, i) v_{\beta_{2}} \cdots u^{\alpha_{1}} \cdots,
$$

where $v_{\alpha}$ are the components of a vector $v \in V(\Lambda)$, while $u^{\alpha}$ are the components of a covector $u \in V^{*}(\Lambda)$. This formula will be useful for constructing 
the invariant actions, since it is the group theory description of the actions used in particle field theories.

It is now also clear how to construct the open spin net functions $\Phi_{\alpha_{1} \ldots}^{\beta_{2} \ldots}\left(g_{e}\right)$. Note that the expansions of the functions over $G^{d}$ used in the field theory reformulations of the $d$-dimensional spin foam amplitudes as Feynman graphs 8, 9, 10, 11, 5], are simply expansions in terms of open spin net functions associated to the $\times_{d}$ graphs ( $d$ edges emanating from a single vertex).

In the case of the BC model [1, 2], the amplitude for the $\times$ graph is not just the intertwiner $C_{\times}^{(i)} \in \operatorname{Inv}\left(\Lambda_{1}, \ldots, \Lambda_{4}\right)$, but it is a linear combination of these

$$
S_{\times}=\sum_{i} \alpha_{i} C_{\times}^{(i)}
$$

This is the consequence of the fact that the subcategory of irreps which are used have invariant vectors under the $S O(3)$ subgroup.

\section{Spin foam amplitudes}

The state sum models which have been used for constructing $d$-dimensional quantum gravity models, for a review see [23, 24], are based on a two-complex $J=(V, E, F)$, consisting of a finite number of vertices $v \in V$, edges $e \in E$ and faces $f \in F$. The two complex $J$ is taken to be dual to a triangulation of the spacetime manifold $M$. Since the faces of $J$ are labeled with the irreps of $G$, one can regard this object as a generalization of the spin net, where the one-complex $\Gamma=(V, E)$ is replaced by a set $(E, F)$ from the two complex $J$. That is why $J$ is called a spin foam [23. Hence these state sum models give the amplitudes for the spin foams. An amplitude for a spin foam can be written as

$$
A(J)=\sum_{\Lambda} \prod_{f \in F} A_{2}\left(\Lambda_{f}\right) \prod_{e \in E} A_{1}\left(\Lambda_{f_{1}(e)} \cdots \Lambda_{f_{k}(e)}\right) \prod_{v \in V} A_{0}\left(\Lambda_{f_{1}(v)} \cdots \Lambda_{f_{l}(v)}\right)
$$

where $A_{2}$ is the amplitude for a face, $A_{1}$ is the amplitude for an edge, and $A_{0}$ is the amplitude for a vertex of the two complex $J$.

There are several ways to determine the amplitudes $A_{n}$, but what is important for our purposes is the fact that these amplitudes are proportional to the amplitudes for the closed spin nets defined in the previous section. In the $d=4$ spacetime case, $A_{0}$ is the amplitude for the 4 -simplex graph (a pentagram) [9, 1, 2]. $A_{1}$ can be one, or it is the amplitude for the $\Theta_{4}$ graph 


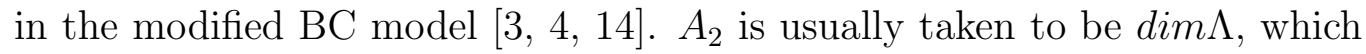
is a single loop spin net amplitude. One can also take $A_{2}$ to be any function of $\Lambda$, such that a group Fourier transform of $A_{2}$ exists [19, 20].

Another aspect of the spin foam amplitude (8) which will be important is the fact that when $A_{2}=\operatorname{dim} \Lambda$ then the amplitude can be represented as a Feynman diagram of a field theory over the group $G[8,9,10,11$, 5]. The action for this field theory can be written in terms of the Fourier modes $\phi$, which transform like tensors from $\operatorname{Inv}\left(\Lambda_{1}, \ldots, \Lambda_{d}\right)$ spaces. In the $d=4$ case the action takes the form

$$
\begin{aligned}
S_{4} & =\frac{1}{2} \sum_{\Lambda, i} Q_{\vec{\alpha}_{1} \vec{\alpha}_{2}}\left(\times_{1}, \times_{2}\right) \phi_{\times_{1}}^{\vec{\alpha}_{1}} \phi_{\times_{2}}^{\vec{\alpha}_{2}} \\
& +\frac{1}{5 !} \sum_{\Lambda, i} \mathcal{V}_{\vec{\alpha}_{1} \cdots \vec{\alpha}_{5}}\left(\times_{1}, \cdots, \times_{5}\right) \phi_{\times_{1}}^{\vec{\alpha}_{1}} \cdots \phi_{\times_{5}}^{\vec{\alpha}_{5}}
\end{aligned}
$$

where $\phi_{\times}^{\vec{\alpha}}=\phi_{\Lambda_{1} \cdots \Lambda_{4}(i)}^{\alpha_{1} \cdots \alpha_{4}}$. The vertex functions $Q$ and $\mathcal{V}$ are given by

$$
\begin{aligned}
Q^{-1}\left(\times_{1}, \times_{2}\right) & =\delta^{4} A_{1}\left(\Lambda_{1}, \cdots, \Lambda_{4} ; i_{1}, i_{2}\right) \\
\mathcal{V}\left(\times_{1}, \cdots, \times_{5}\right) & =\delta^{10} A_{0}\left(\Lambda_{1}, \cdots, \Lambda_{10} ; i_{1}, \ldots, i_{5}\right)
\end{aligned}
$$

where $\delta$ are the appropriate delta functions of the indices. $A_{1}$ can be the amplitude for the $\Theta_{4}$ graph, or it could be one, when is the amplitude for four parallel lines. $A_{0}$ is the amplitude for the pentagram. In the case of $\mathrm{BC}$ models, $A_{0}$ and $A_{1}$ will not depend on the intertwiner labels $i_{k}$.

The corresponding Feynman diagram is just the one complex $(V, E)$ obtained from the two complex $J=(V, E, F)$. This diagram is a $(d+1)$-valent graph in the case of $d$-dimensional model. Its value is given by (8), which can be represented by a collection of spin net diagrams, such that to each vertex we associate a $d$-simplex graph, while to each edge we associate $d$ parallel lines, or a $\Theta_{d}$ graph in the modified version.

\section{Fermionic matter}

The Fourier modes $\phi_{\times}^{\vec{\alpha}}$ can be promoted into creation and annihilation operators [5], so that the spin foam amplitude can be represented as an matrix element of an evolution operator, in analogy with the particle field theory case. As shown in [5], one can construct the "in" and the "out" states which 
describe the spatial spin nets corresponding to the boundary triangulations of the spacetime manifold. Such spin net states can be represented by

$$
|\gamma\rangle=\prod_{v \in V(\gamma)} \phi_{\times_{v}}^{\dagger}|0\rangle
$$

where $|0\rangle$ is the vacuum of the Fock space constructed from the creation and annihilation operators $\phi$ and $\phi^{\dagger}$, and $\gamma$ is a four-valent spin net graph dual to the boundary triangulation. All the representation indices $\alpha_{v}$ in (12) are appropriately contracted.

In the loop gravity formalism [21, 22], the fermionic matter is introduced by replacing the spin net $\gamma$ with an open spin net $\gamma_{F}$, which is obtained from $\gamma$ by putting an external edge carrying a fermionic irrep $\Lambda_{s}$ at each site of $\gamma$ where a fermion is located. One can also put more than one fermion at each lattice site, up to $\operatorname{dim} \Lambda_{s}$ (Pauli exclusion principle). In that case, the external edge is labeled with an irrep from an antisymmetrized tensor product of $k \Lambda_{s}$ irreps [22].

In the spin foam formalism, the analogue of the loop gravity construction would be to construct the $\left|\gamma_{F}\right\rangle$ state by introducing the fermionic creation and annihilation operators $\psi_{\sigma}\left(\times_{v}\right)$ and $\psi_{\sigma}^{\dagger}\left(\times_{v}\right)$, where $\sigma$ is the representation index of $\Lambda_{s}$, and the label $\times_{v}$ denotes the four irreps of the spin net site where the fermion is located. Hence the corresponding state would be given as

$$
\left|\gamma_{F}\right\rangle=\prod_{v^{\prime} \in V^{\prime}(\gamma)} \prod_{\sigma_{v^{\prime}}} \psi_{\sigma_{v^{\prime}}}^{\dagger}\left(\times_{v^{\prime}}\right) \prod_{v \in V(\gamma)} \phi_{\times_{v}}^{\dagger}|0\rangle
$$

where $V^{\prime}$ is the set of vertices where the fermions are located.

In order to calculate the transition amplitudes between two fermionic spin net states, which would be a fermionic spin foam amplitude, we need to introduce the corresponding terms in the field theory action (9). Since classically the fermionic propagation is described by a line in the spacetime, and since the fermions interact with the gravitational field, the candidate action will be

$$
S_{F}=\sum_{\Lambda} \psi^{\sigma}(\times) \psi^{\sigma^{\prime}}\left(\times^{\prime}\right) \mathcal{V}_{\sigma \sigma^{\prime}}\left(\times, \times^{\prime}, \times_{1}, \cdots, \times_{n}\right) \phi_{\times_{1}} \cdots \phi_{\times_{n}}+(\text { h.c. })
$$

where (h.c.) stands for the hermitian conjugate term.

The form of the vertex $\mathcal{V}$ can be restricted by two requirements. First, $S_{F}$ has to be a group invariant, i.e. a scalar. Second, we require that the fermions 
propagate only on complexes which are dual to the Feynman graphs of the gravitational model (9). The idea behind this is that the purely gravitational model builds up the space-time on which the fermions propagate. Hence $n=5$, and

$$
\mathcal{V}_{\sigma \sigma^{\prime}}\left(\times, \times^{\prime}, \times_{1}, \cdots, \times_{5}\right)=\delta^{10} \delta_{\times, \times_{1}} \delta_{\times^{\prime}, \times_{k}} A_{\sigma \sigma^{\prime}}\left(\Lambda_{1}, \ldots, \Lambda_{10}\right)
$$

where $A_{\sigma \sigma^{\prime}}$ is a spin net amplitude for the pentagram with two external edges attached at site 1 and site $k$, where $1 \leq k \leq 5$.

Note that one can take a more complicated spin net graph for the amplitude $A_{\sigma \sigma^{\prime}}$, by adding internal edges labelled by the matter irrep, see sect. 5 . One would then need to study the semiclassical limit in order to determine which spin net would give a correct physical theory. Since that is beyond the scope of this paper, and for the sake of simplicity, we will analyze only the simplest possible spin nets.

We will also add to $S_{F}$ a purely quadratic fermion term

$$
\sum_{\Lambda} \psi^{\sigma}(\times) Q_{\sigma \sigma^{\prime}}\left(\times, \times^{\prime}\right) \psi^{\sigma^{\prime}}\left(\times^{\prime}\right)+(\text { h.c. })
$$

in order to have a well-defined perturbative expansion. The propagator $Q^{-1}$ will be determined by the amplitude for the $\Theta_{4}$ graph with two external edges labelled with $\Lambda_{s}$, or it could be a trivial amplitude for five parallel lines. We denote the propagator amplitude as $G_{\sigma \sigma^{\prime}}$.

Note that $\left(\psi_{\sigma}\right)^{*}=\psi^{\sigma}=C^{\sigma \sigma^{\prime}} \psi_{\sigma^{\prime}}$, and the vector space duality $*$ is in general different from the complex conjugation (reality) properties. The reality properties determine relation between the creation and annihilation operators $\psi$ and $\psi^{\dagger}[0]$.

Therefore the maximal closed spin net subgraph for $A_{\sigma \sigma^{\prime}}$ has to be at least the pentagram graph. Hence a relevant fermionic Feynman diagram $\Gamma_{F}$ which is generated by the $S_{4}+S_{F}$ action will be determined by a spacetime skeleton diagram $\Gamma$ (a 5-valent graph dual to a spacetime triangulation) plus a line connecting the vertices of $\Gamma$. Therefore $\Gamma_{F}$ will be a graph consisting of 5 -valent and 7 -valent vertices, see Fig. 1. Out of these diagrams we will consider only those for which the fermion path is a line which connects the centers of a string of adjacent 4-simplices, extending from the initial to the final boundary.

Hence the fermionic spin foam $J_{F}$ will be given by the usual spin foam $J$ and a line $L$ of edges starting from an "in" edge and terminating with an 
"out" edge. The corresponding amplitude will be given by

$$
A_{\sigma_{i}}^{\sigma_{f}}\left(J_{F}\right)=\sum_{\Lambda} \prod_{f \in F} A_{f}(\Lambda) A_{\sigma_{i}}^{\sigma_{f}}(L) \prod_{e \in E^{\prime}} A_{e}(\Lambda) \prod_{v \in V^{\prime}} A_{v}(\Lambda)
$$

where

$$
A_{\sigma_{i}}^{\sigma_{f}}(L)=A_{\sigma_{i}}^{\sigma_{1}}\left(v_{1}\right) G_{\sigma_{1}}^{\sigma_{2}}\left(e_{12}\right) A_{\sigma_{2}}^{\sigma_{3}}\left(v_{2}\right) \cdots G_{\sigma_{k-1}}^{\sigma_{k}}\left(e_{k-1, k}\right) A_{\sigma_{k}}^{\sigma_{f}}\left(v_{k}\right)
$$

The number of the "in" and the "out" fermions has to be the same for a free theory, and the above formula can be then easily generalized to the case when there are several lines $L$.

The amplitude $A$ for a fermionic vertex of $J_{F}$ will be given by a spin net amplitude for a pentagram with two external fermionic edges, Fig. 2

$$
A_{\sigma^{\prime}}^{\sigma}\left(\Lambda_{1}, \ldots, \Lambda_{10}\right)=\sum_{\alpha} C_{\sigma^{\prime} \times} C_{\times}^{\sigma} C_{\times} C_{\times} C_{\times}
$$

where $C_{\sigma^{\prime} \times}, C_{\times}^{\sigma} \in \operatorname{Inv}\left(\Lambda_{s}, \Lambda_{1}, \ldots, \Lambda_{4}\right)$ and $C_{\times} \in \operatorname{Inv}\left(\Lambda_{1}, \ldots, \Lambda_{4}\right)$. The amplitude for an fermionic edge of $J_{F}$ will be given by the propagator amplitude $G_{\sigma}^{\sigma^{\prime}}$, which in the non-trivial case is given by the amplitude for the $\Theta_{4}$ graph with two external edges, Fig. 3

$$
G_{\sigma^{\prime}}^{\sigma}\left(\Lambda_{1}, \ldots, \Lambda_{4}\right)=\sum_{\alpha} C_{\sigma^{\prime} \times} C_{\times}^{\sigma}
$$

In some of the cases the minimal amplitudes (19) and (20) are zero, because $C_{\sigma \times}=0$. Then one can replace it with $C_{\sigma \sigma^{\prime} \times}$ from $\operatorname{Inv}\left(\Lambda_{s}, \Lambda_{s}, \Lambda_{1}, \ldots, \Lambda_{4}\right)$, so that

$$
A_{\sigma^{\prime}}^{\sigma}\left(\Lambda_{1}, \ldots, \Lambda_{10}\right)=\sum_{\alpha} C_{\sigma^{\prime} \times}^{\tau} C_{\tau \times}^{\sigma} C_{\times} C_{\times} C_{\times} .
$$

The corresponding spin net graph is a pentagram with two external and one internal fermion edge, joined in a line, Fig. 4. Similarly, for the edge amplitude $G_{\sigma}^{\sigma^{\prime}}$, we will have in the non-trivial case the $\Theta_{5}$ graph with two external edges, Fig. 5, so that

$$
G_{\sigma^{\prime}}^{\sigma}\left(\Lambda_{1}, \ldots, \Lambda_{4}\right)=\sum_{\alpha} C_{\sigma^{\prime} \tau \times} C_{\times}^{\tau \sigma}
$$




\section{Euclidian case}

We now study the four-dimensional Euclidian gravity spin foam model [1], 3], so that $G=S O(4)$. The irreps of $S O(4)$ can be labeled as $\Lambda=(j, k)$ where $j$ and $k$ are the half-integers labelling the irreps of $S U(2)$. In the topological gravity case, one uses all $S O(4)$ irreps for labelling the triangles of a triangulation of $M$. The fermions will be represented by $\Lambda_{s}^{+}=\left(\frac{1}{2}, 0\right)$ and $\Lambda_{s}^{-}=\left(0, \frac{1}{2}\right)$ irreps. These irreps correspond to chiral, or Weyl fermions, while a Dirac fermion would be a reducible representation $\left(\frac{1}{2}, 0\right) \oplus\left(0, \frac{1}{2}\right)$.

The relevant intertwiners for constructing the fermionic spin foam amplitude are from the space $\operatorname{Inv}\left(\Lambda_{s}, \Lambda_{1}, \ldots, \Lambda_{4}\right)$. A basis $|i\rangle$ is determined by

$$
C_{\sigma \alpha_{1} \ldots \alpha_{4}}^{\Lambda_{s} \Lambda_{1} \ldots \Lambda_{4}(i)}=\sum_{\alpha, \alpha^{\prime}} C_{\sigma \alpha_{1} \alpha}^{\Lambda_{s} \Lambda_{1} \Lambda} C_{\alpha \alpha_{2} \alpha^{\prime}}^{\Lambda \Lambda_{2} \Lambda^{\prime}} C_{\alpha^{\prime} \alpha_{3} \alpha_{4}}^{\Lambda^{\prime} \Lambda_{3} \Lambda_{4}}
$$

In order to simplify the notation, we take $X_{\alpha} Y_{\alpha}=X^{\alpha} Y_{\alpha}=C^{\alpha \beta} X_{\alpha} Y_{\beta}$. Note that the formula $(23)$ can be represented graphically by Fig. 6. Hence the intertwiner label is given by $i=\left(\Lambda, \Lambda^{\prime}\right)$, and we can now construct the fermionic amplitudes for topological gravity spin foam model by using the procedure described in sect. 4. However, we will not discuss further the topological gravity case, and we will concentrate on the non-topological model, because of its importance for physics.

The set of relevant irreps in the non-topological gravity case is given by the simple irreps $N=(j, j)$. In that case $C_{\ldots \ldots}^{\Lambda_{s} N_{1} \ldots N_{4}}=0$, which can be proven by using a $Z_{2}$ grading of the $S O(4)$ irreps. This grading is introduced by splitting the irreps into even and odd, or bosonic and fermionic irreps via the parity function $p(n)=\{-1,1\}$ if $n$ is odd, even, respectively, as

$$
p(j, k)=p(2 j) p(2 k) \text {. }
$$

Hence the simple irreps are even, i.e. bosonic, while $\Lambda_{s}^{ \pm}$are odd, i.e. fermionic. Since $p\left(\Lambda_{1} \otimes \Lambda_{2}\right)=p\left(\Lambda_{1}\right) p\left(\Lambda_{2}\right)=p(\Lambda)$ where $\Lambda$ is an irrep from the tensor product, then a product of bosonic irreps can never yield a fermionic irrep, and hence $C^{\Lambda_{s} N_{1} \ldots N_{4}}=0$. Therefore the minimal option for the intertwiner space is $\operatorname{Inv}\left(\Lambda_{s}, \Lambda_{s}, N_{1}, \ldots, N_{4}\right)$, and a basis $|i\rangle$ can be constructed from

$$
C_{\sigma \sigma^{\prime} \alpha_{1} \ldots \alpha_{4}}^{\Lambda_{s} \Lambda_{s} N_{1} \ldots N_{4}(i)}=\sum_{\beta_{1}, \beta_{2}, \beta_{3}} C_{\sigma \sigma^{\prime} \beta_{1}}^{\Lambda_{s} \Lambda_{s} B_{1}} C_{\alpha_{1} \alpha_{2} \beta_{2}}^{N_{1} N_{2} B_{2}} C_{\beta_{1} \beta_{2} \beta_{3}}^{B_{1} B_{2} B_{3}} C_{\beta_{3} \alpha_{3} \alpha_{4}}^{B_{3} N_{3} N_{4}}
$$

where $B$ are the bosonic irreps. The equation (25) can be graphically represented by Fig. 7 . 
Now we have to impose the constraints coming from the fact that the simple irrep vector spaces have invariant vectors under the $S O(3) \cong S U(2)$ subgroup. This amounts to constructing an invariant vector in the corresponding $\operatorname{Inv}(n)$ space. When there are no fermions, then $n=4$, and the invariant vector, or the $\mathrm{BC}$ vertex, is constructed as [1]

$$
S_{\alpha_{1} \ldots \alpha_{4}}^{N_{1} \ldots N_{4}}=\sum_{M} C_{\alpha_{1} \ldots \alpha_{4}}^{N_{1} \ldots N_{4}(M)}=\sum_{M} \sum_{\alpha} C_{\alpha_{1} \alpha_{2} \alpha}^{N_{1} N_{2} M} C_{\alpha \alpha_{3} \alpha_{4}}^{M N_{3} N_{4}},
$$

where $M$ are the simple irreps. The $3 j$ symbols for $S O(4)$ can be expressed as the product of $3 j$ symbols for $S U(2)$ as

$$
C_{\alpha_{1} \alpha_{2} \alpha_{3}}^{N_{1} N_{2} N_{3}}=C_{m_{1} m_{2} m_{3}}^{j_{1} j_{2} j_{3}} C_{n_{1} n_{2} n_{3}}^{j_{1} j_{2} j_{3}}
$$

The consequence of using the $B C$ vertex for constructing the spin net amplitudes is that these amplitudes can be represented as multiple integrals over the coset space $X=G / H$, which is a three-sphere $S^{3}=S O(4) / S O(3)$ [25, 26]. For example, the amplitude for the $\Theta_{4}$ graph will be given by

$$
\Theta\left(N_{1}, \ldots, N_{4}\right)=\sum_{\alpha} S_{\alpha_{1} \ldots \alpha_{4}}^{N_{1} \ldots N_{4}} S_{\alpha_{1} \ldots \alpha_{4}}^{N_{1} \ldots N_{4}}
$$

One can show that

$$
\Theta\left(N_{1}, \ldots, N_{4}\right)=\int_{X^{2}} d x d y \prod_{i=1}^{4} K_{N_{i}}(x, y)
$$

where

$$
K_{N}(x, y)=\operatorname{Tr} D^{(j)}\left(x^{-1} \cdot y\right)=\frac{\sin ((2 j+1) \theta)}{\sin \theta},
$$

and $\theta$ is the geodesic distance between the coset points. This formula works because the coset points $x$ and $y$ can be considered as $S U(2)$ group elements due to equivalence of $S^{3}$ and $S U(2)$ spaces.

We will now use this property to construct the fermionic BC vertex. Consider the following integral associated to the $\Theta_{4}$ graph

$$
\Theta_{s s^{\prime}}\left(N_{1}, \ldots, N_{4}\right)=\int_{X^{2}} d x d y K_{s s^{\prime}}(x, y) \prod_{i=1}^{4} K_{N_{i}}(x, y),
$$

where

$$
K_{s s^{\prime}}(x, y)=D_{s s^{\prime}}^{(1 / 2)}\left(x^{-1} \cdot y\right)
$$


One can now show that

$$
\Theta_{s s^{\prime}}\left(N_{1}, \ldots, N_{4}\right)=\sum_{\alpha, \tilde{s}} S_{\alpha_{1} \ldots \alpha_{4} \tilde{s} s}^{N_{1} \ldots N_{4} N_{1 / 2}} S_{\alpha_{1} \ldots \alpha_{4} \tilde{s} s^{\prime}}^{N_{1} \ldots N_{4} N_{1 / 2}}
$$

where $S$ is an element of $\operatorname{Inv}\left(N_{1}, \ldots, N_{4}, N_{1 / 2}\right)$ given by

$$
S_{\alpha_{1} \ldots \alpha_{4} \sigma}^{N_{1} \ldots N_{4} N_{1 / 2}}=\sum_{M, N} \sum_{\alpha, \beta} C_{\alpha_{1} \alpha_{2} \alpha}^{N_{1} N_{2} M} C_{\alpha \sigma \beta}^{M N_{1 / 2} N} C_{\beta \alpha_{3} \alpha_{4}}^{N N_{3} N_{4}}
$$

and $N_{1 / 2}=\left(\frac{1}{2}, \frac{1}{2}\right)$.

When (34) is compared to (25), then it is easy to see that the expression (34) can be also taken as an intertwiner from the space $\operatorname{Inv}\left(\Lambda_{s}^{+}, \Lambda_{s}^{-}, N_{1}, \ldots, N_{4}\right)$, due to $N_{1 / 2}=\Lambda_{s}^{+} \otimes \Lambda_{s}^{-}$. Therefore the expression (31) can be interpreted as the amplitude for the open spin net consisting of the $\Theta_{4}$ graph plus two external edges labelled with $\Lambda_{s}^{+}$and one internal edge labeled with $\Lambda_{s}^{-}$, Fig. 5 .

Hence the action $S_{F}$ for the $\Lambda_{s}$ fermions will be given by

$$
\begin{aligned}
S_{F} & =\sum_{N} \psi_{ \pm}^{\sigma}(\times) Q_{\sigma \nu}\left(\times, \times^{\prime}\right) \psi_{ \pm}^{\nu}\left(\times^{\prime}\right) \\
& +\sum_{N} \psi_{ \pm}^{\sigma}(\times) \psi_{ \pm}^{\nu}\left(\times^{\prime}\right) \mathcal{V}_{\sigma \nu}\left(\times, \times^{\prime} ; \times_{1} \cdots \times_{5}\right) \phi_{\times_{1}} \cdots \phi_{\times_{5}} \\
& + \text { (h.c. })
\end{aligned}
$$

where $Q^{-1}$ and $\mathcal{V}$ are determined by the

$$
G_{\sigma \nu}\left(N_{1} \ldots N_{4}\right)=\int_{X^{2}} d^{2} x K_{\sigma \nu}\left(x_{1}, x_{2}\right) \prod_{i} K_{N_{i}}\left(x_{1}, x_{2}\right)
$$

and

$$
A_{\sigma \nu}\left(N_{1} \ldots N_{10}\right)=\int_{X^{5}} d^{5} x K_{\sigma \nu}\left(x_{1}, x_{k}\right) \prod_{i<j} K_{N(i j)}\left(x_{i}, x_{j}\right)
$$

spin net amplitudes, respectively. The edge amplitude (36) is determined by the graph from Fig. 5, and the vertex amplitude (37) is determined by the graph from Fig. 4.

The reason why (31) and (33) are the same is that the spinor propagator (32) is an example of a matrix spherical function [27], which is a generalization of the scalar propagator $K_{N}$ (30). In general case [27], if $H$ is a subgroup 
of $G$ and if $\tau$ is an irrep of $H$, consider irreps $\Lambda$ of $G$ which contain $\tau$ when decomposed with respect to the subgroup $H$, i.e.

$$
V_{\Lambda}=\bigoplus_{1 \leq k \leq n} V_{\tau}^{k} \oplus \ldots=H_{\tau} \oplus \ldots
$$

Let $\Phi(g)=P_{\tau} D^{(\Lambda)}(g) P_{\tau}$, where $P_{\tau}$ is the projector from $V_{\Lambda}$ to $H_{\tau}$. $\Phi(g)$ are called $\tau$-spherical functions, and $P_{\tau}^{k} D^{(\Lambda)}(g)$ are eigenfunctions of the Laplacian on the homogenous vector bundle $E_{\tau}=\left(G / H, V_{\tau}\right)$. Since $\Phi(g) \in$ $\operatorname{Hom}\left(H_{\tau}, H_{\tau}\right)$, we can construct $\phi(g)=\operatorname{tr} \Phi(g) \in \operatorname{Hom}\left(V_{\tau}, V_{\tau}\right)$, where $\operatorname{tr}$ is a partial trace over the degeneracy label $k$. Then consider the object

$$
K_{\Lambda, \tau}(x, y)=U\left(x, x_{0}\right) \phi\left(g_{x}^{-1} g_{y}\right) U\left(x_{0}, y\right)
$$

where $U$ 's are vector bundle parallel transport operators along the geodesics $\left(x_{0}, x\right)$ and $\left(x_{0}, y\right)$, and $g_{x}=\sigma(x)$ is a local section of the principal bundle $(G / H, G)$. By using $g_{x}=x h, g_{y}=y \tilde{h}, U\left(x, x_{0}\right)=D^{(\tau)}(h)$ and $U\left(x_{0}, y\right)=$ $D^{(\tau)}\left(\tilde{h}^{-1}\right)$, one can show that $K$ is a function of only the coset space points. Note that when $\tau$ is the identity irrep, the expression (39) reduces to the scalar propagator $K_{N}$, where $N$ is the class one irrep. Hence (39) is the matrix generalization of $K_{N}$, and we can use it to construct the spin net amplitudes for graphs with external edges.

In the $S O(4)$ case, let $\overrightarrow{\mathcal{A}}$ and $\overrightarrow{\mathcal{B}}$ be the generators of the two commuting $S U(2)$. Then the $S O(4)$ rotations generators $J_{\mu \nu}$ can be expressed as

$$
\vec{J}=\overrightarrow{\mathcal{A}}+\overrightarrow{\mathcal{B}} \quad, \quad \vec{K}=\overrightarrow{\mathcal{A}}-\overrightarrow{\mathcal{B}}
$$

where $J_{i}=\epsilon_{i}{ }^{j k} J_{j k}$ and $K_{i}=J_{i 0}$. The $S O(4)$ irreps $\Lambda$ are then determined by the pairs of half-integers $(A, B)$, where $A$ and $B$ are the angular momenta numbers of the two $S U(2)$. We then have

$$
\begin{aligned}
D^{(A, B)}(\vec{\theta}, \vec{v}) & =\exp \left(i \vec{\theta} \vec{J}_{\Lambda}+i \vec{v} \vec{K}_{\Lambda}\right) \\
& =\exp \left(i \vec{\theta}_{+} \overrightarrow{\mathcal{A}}_{A}+i \vec{\theta}_{-} \overrightarrow{\mathcal{B}}_{B}\right) \\
& =D^{(A)}\left(\vec{\theta}_{+}\right) \otimes D^{(B)}\left(\vec{\theta}_{-}\right)
\end{aligned}
$$

where

$$
\vec{\theta}_{ \pm}=\vec{\theta} \pm \vec{v}
$$

The diagonal $S U(2)$ subgroup vectors are given by

$$
|j, m\rangle=C_{m a b}^{j A B}|a\rangle \otimes|b\rangle
$$


These vectors form an irrep space of the spatial rotation generators $\vec{J}$. In the case of $\Lambda_{s}$, we take $\tau=j=1 / 2$, so that $P_{\tau}=1$. From (41) we get

$$
K_{1 / 2}^{E}(x, y)=D^{(1 / 2)}\left(x^{-1} \cdot y\right)=D^{(1 / 2)}(-\vec{v}) D^{(1 / 2)}(\vec{u})
$$

where

$$
D^{(1 / 2)}(\vec{r})=\cos (r / 2) I_{2}+i \vec{\sigma} \vec{n} \sin (r / 2) \quad, \quad \vec{n}=\frac{\vec{r}}{\sqrt{(\vec{r})^{2}}}=\frac{\vec{r}}{r},
$$

and $\vec{\sigma}$ are the Pauli matrices.

\section{Lorentzian case}

In the Lorentzian case $G=S O(3,1) \cong S L(2, C)$, and the finite-dimensional irreps $(j, k)$ become pseudo unitary, because the relation (40) becomes

$$
\vec{J}=\overrightarrow{\mathcal{A}}+\overrightarrow{\mathcal{B}} \quad, \quad \vec{K}=(-i)(\overrightarrow{\mathcal{A}}-\overrightarrow{\mathcal{B}})
$$

so that the boost generator $\vec{K}$ becomes an anti-hermitian operator. This is the reason why there are no finite-dimensional unitary irreps for the Lorentz group.

Hence the unitary Lorentz irreps are infinite-dimensional, and can be labelled as $(m, \rho)$, where $m$ is a half-intiger, while $\rho$ is a non-negative real number [?]. The gravitational DOF are again described by the simple irreps, or the class one irreps with respect to the spatial $S U(2)$ subgroup. These are given by the $(m, 0)$ and $(0, \rho)$ irreps [2]. However, only the $(0, \rho)$ irreps are used [2]. We could then use the pseudo unitary irreps $(j, k)$ to describe the matter fields, but we have to check first the consistency of the state-sum model with unitary and pseudo unitary irreps of the Lorentz group.

We now have $N=(0, \rho)$ in the gravitational sector. The corresponding propagator is given by [2]

$$
K_{\rho}(x, y)=\frac{\sin (\rho d(x, y))}{\rho \sinh d(x, y)}
$$

where $d(x, y)$ is the geodesic distance between the points $x$ and $y$ of the coset space $X$, which is now a hyperboloid $S O(1,3) / S O(3)$. The fermions will carry the irreps $\Lambda_{s}^{ \pm}$. The tensor product decomposition rule of a unitary and 
a pseudo-unitary irrep can be inferred from the decomposition of these irreps under the $S U(2)$ subgroup. For the $(m, \rho)$ irrep we have [28]

$$
\mathcal{H}_{(m, \rho)}=\bigoplus_{j=|m|}^{\infty} V_{j} .
$$

Since $V\left(\Lambda_{s}^{ \pm}\right)=V_{\frac{1}{2}}$, we obtain

$$
\mathcal{H}_{(m, \rho)} \otimes V\left(\Lambda_{s}^{ \pm}\right)=\mathcal{H}_{\left(m-\frac{1}{2}, \rho\right)} \oplus \mathcal{H}_{\left(m+\frac{1}{2}, \rho\right)},
$$

which agrees with 15. This implies

$$
\mathcal{H}_{(m, \rho)} \otimes V\left(\Lambda_{v}\right)=\mathcal{H}_{(m-1, \rho)} \oplus \mathcal{H}_{(m, \rho)} \oplus \mathcal{H}_{(m, \rho)} \oplus \mathcal{H}_{(m+1, \rho)}
$$

where $\Lambda_{v}=\left(\frac{1}{2}, \frac{1}{2}\right)$.

Hence $C^{\rho_{1} \rho_{2} \Lambda_{v}} \neq 0$, and we can now construct the Lorentzian analog of the $\mathrm{BC}$ spinor vertex (34). For this we need a Lorentzian spinor propagator, which is determined by the formula (39). It is given by an analytic continuation of the Euclidian formula (44), where $\vec{v} \rightarrow-i \vec{v}$, so that

$$
K_{1 / 2}^{L}(x, y)=D^{(1 / 2)}\left(x^{-1} \cdot y\right)=D^{(1 / 2)}(i \vec{v}) D^{(1 / 2)}(-i \vec{u})
$$

where now

$$
D^{(1 / 2)}(i \vec{r})=\cosh (r / 2) I_{2}+\vec{\sigma} \vec{n} \sinh (r / 2)
$$

\section{Interactions and gauge fields}

Note that in the discussion in section 4 one could have taken $\Lambda_{s}$ to be an arbitrary irrep of $G$, and moreover, one can take a set of different irreps $\Lambda_{s_{1}}=S_{1}, \ldots, \Lambda_{s_{k}}=S_{k}$ to represent fields of different spins. In the topological gravity case, one can write the action as

$$
\begin{aligned}
S_{I} & =\sum_{S} \sum_{\Lambda} \psi_{S}(\times) \psi_{S}\left(\times^{\prime}\right) Q_{S S}\left(\times, \times^{\prime}\right) \\
& +\sum_{S} \sum_{\Lambda} \psi_{S}(\times) \psi_{S}\left(\times^{\prime}\right) \mathcal{V}_{S S}\left(\times, \times^{\prime}, \times_{1}, \cdots, \times_{5}\right) \phi_{\times_{1}} \cdots \phi_{\times_{5}} \\
& +\sum_{S} \sum_{\Lambda} \psi_{S_{1}}(\times) \cdots \psi_{S_{k}}\left(\times^{(k)}\right) \mathcal{V}_{S_{1} \ldots S_{k}}\left(\times, \ldots, \times^{(k)} ; \times_{1}, \ldots, \times_{5}\right) \phi_{\times_{1}} \cdots \phi_{\times_{5}} \\
& +(\text { h.c. })
\end{aligned}
$$


where the vertex $Q$ will be determined by the spin net amplitudes for the open graphs based on the five parallel lines and the $\Theta_{4}$, while the vertex $\mathcal{V}$ will be determined by the spin net amplitudes for open graphs based on the pentagram. A new feature is that the set of possible graphs for the vertex $\mathcal{V}_{S_{1} \ldots S_{k}}$ will contain graphs with more than five vertices, Fig. 8.

In the non-topological gravity case, we need to implement the $H$ invariance. This requires a specification of the irreps $s$ of $H$ which are contained in the irreps $S$ of $G$. In that case the matter fields will have the indices from the $H$ irreps, and the action can be written as

$$
\begin{aligned}
S_{I} & =\sum_{S} \sum_{N} \psi_{s}(\times) \psi_{s}\left(\times^{\prime}\right) Q_{s s}^{S}\left(\times, \times^{\prime}\right) \\
& +\sum_{S} \sum_{N} \psi_{s}(\times) \psi_{s}\left(\times^{\prime}\right) \mathcal{V}_{s s}^{S}\left(\times, \times^{\prime}, \times_{1}, \cdots, \times_{5}\right) \phi_{\times_{1}} \cdots \phi_{\times_{5}} \\
& +\sum_{S} \sum_{N} \psi_{s_{1}}(\times) \cdots \psi_{s_{k}}\left(\times^{(k)}\right) \mathcal{V}_{s_{1} \ldots s_{k}}^{S_{1} \ldots S_{k}}\left(\times, \ldots, \times^{(k)} ; \times_{1}, \ldots, \times_{5}\right) \phi_{\times_{1}} \cdots \phi_{\times_{5}} \\
& +(h . c)
\end{aligned}
$$

where the vertices $Q$ and $\mathcal{V}$ will be determined by the spin net amplitudes for open graphs based on five parallel lines, the $\Theta_{4}$ and the pentagram graphs, as in the topological case. However, these amplitudes will be now given as multiple integrals of the propagators $K_{N}(x, y)$ and $K_{S, s}(x, y)$. Also note that the fields $\psi_{s}$ will now carry the indices from the irreps of the subgroup $H$.

In the Euclidian/Lorentzian gravity case $H=S O(3)$, and it will be instructive to study the case of spin $j=1$ matter fields, because in the nature the fermions interact through the spin $j=1$ gauge bosons. If we take a vector irrep $S_{1}=\left(\frac{1}{2}, \frac{1}{2}\right)$, it will contain a $s=j=1$ irrep of $S O(3)$. We denote the corresponding spin net field as $A_{i}(\times)$, while the fermions we denote as $\psi_{\sigma}^{ \pm}(\times)=\psi_{s}^{ \pm}(\times)$, where the subscripts \pm denote the corresponding $S O(4)$ irreps. The spin foam analog of the particle field theory interaction term $\int d^{4} x \bar{\psi} \gamma^{\mu} \psi A_{\mu}$ can be written as

$$
\sum_{N} \psi_{s}^{+}(\times) \psi_{s^{\prime}}^{-}\left(\times^{\prime}\right) A_{i}\left(\times^{\prime \prime}\right) \mathcal{V}_{+-}^{s s^{\prime} i}\left(\times, \times^{\prime}, \times^{\prime \prime} ; \times_{1}, \ldots, \times_{5}\right) \phi_{\times_{1}} \cdots \phi_{\times_{5}}+(\text { h.c. }),
$$

where the vertex $\mathcal{V}_{+-}$is determined by the spin net amplitude for the open graph given by the pentagram plus a three-valent matter vertex, Fig. 9. This 
amplitude is given by

$$
A_{+-}^{s s^{\prime} i}=\int_{X^{6}} d^{5} x d y K_{\frac{1}{2}}^{s t}\left(x_{1}, y\right) K_{\left(\frac{1}{2}, \frac{1}{2}\right), 1}^{i j}\left(x_{4}, y\right) K_{\frac{1}{2}}^{s^{\prime} t^{\prime}}\left(x_{2}, y\right) C_{t t^{\prime} j}^{\frac{1}{2} \frac{1}{2} 1} \prod_{k<l} K_{N_{k l}}\left(x_{k}, x_{l}\right)
$$

Since (55) is a spin foam generalization of the particle gauge field theory $U(1)$ interaction, it is natural to ask what is the analog of the $U(1)$ gauge symmetry. One way to deal with the issue of gauge symmetry is to assume that the components of the fields appearing in the action (54) are the physical, or the independent DOF. Note that one can adopt this type of approach in the context of flat space particle field theory [17]. One simply takes the creation and annihilation operators labelled by the irreducible Poincare representation indices and then forms polynomial interaction terms via the formula (6). The relevant Poincare irreps are the massive and the massless ones, so that the index labels are the three-momentum, the $S O(3)$ irrep indices for the massive case, or the two-dimensional $\operatorname{Spin}(2) \cong S O(2)$ representation labels in the massless case.

Since one wants the particle interactions to be local in spacetime, one then constructs spacetime fields carrying the appropriate Lorentz irreps from the Poncare creation and annihilation (c/a) operators, and writes the interactions as spacetime integrals over the polynomials of these fields [17]. The problem with this approach is that the massless fields cannot be always expressed in terms of the helicity c/a operators. This happens for the vector field $A_{\mu}(x)$, which when expressed in terms of the helicity c/a operators transforms nonhomogenously under the Lorentz transformations [17]. However, the field $f_{\mu \nu}=\partial_{[\mu} A_{\nu]}$ transforms like a Lorentz tensor, so that the cubic interaction term would be given by $\int d^{4} x \psi \gamma^{\mu \nu} \psi f_{\mu \nu}$. But this is not the type of interaction realized in nature, which is $\int d^{4} x \bar{\psi} \gamma_{\mu} \psi A^{\mu}$. This paradox is resolved by introducing the $U(1)$ gauge transformations [17].

In the case of our spin foam model, this type of problem will not appear immediately, because one is dealing only with the $\mathrm{c} / \mathrm{a}$ operators for the Lorentz group and its subgroups. These operators carry the labels $\times=\left(N_{1} \ldots N_{4}\right)$ and $S U(2) \cong S O(3)$ representation indices. There are no Poincare irreps labels, and the reason for that is that the particle momentum is not conserved in curved spacetimes. Hence it is not clear what would be a physical interpretation of a spin-foam model based on the Poincare group

\footnotetext{
†This can be seen on the example of a Poincare spin foam model based on the simple
} 
Our assumption that the components of the spin net fields are the physical components can be further justified by the fact that these fields live on the spin nets defined by the triangulations of three-space boundaries, see equation (13). We will then consider these fields as the discretized analogs of the reduced phase space canonical formalism fields. The canonical analysis of the continuous gravity and matter actions shows that the effect of the local Lorentz and the spacetime diffeomorphism constraints is to reduce the number of components of the gravitational field, while the matter field components are affected only by the gauge symmetry constraints. The final result is that the non-gauge fields have independent components which transform as the appropriate representations of $S O(3)$, while the gauge fields independent components transform as the appropriate representations of $S O(2)$. In the flat space limit, these independent component fields become the Fourier transforms of the Poincare irreps, so that the non-gauge fields correspond to the massive irreps, while the gauge fields correspond to the massless ones 3 .

Therefore within this approach the $A_{i}(\times)$ operator cannot be considered as a discrete massless gauge field analog, since it carries a $j=1$ irrep of $S O(3)$, which corresponds to a massive Poincare irrep. In order to introduce a massless-like vector gauge boson in the model, we need a spin net field which transforms as a two-dimensional representation of $S O(2)$. Therefore we need to construct invariant propagators under an $S O(2)$ subgroup of the $S O(3)$ subgroup.

Hence consider a subgroup $H^{\prime}$ of $H$, and let $\tau^{\prime}$ be a representation (could be reducible) of $H^{\prime}$ contained in the irrep $\tau$ of $H$. Note that one cannot use the $K_{\Lambda, \tau^{\prime}}$ propagators for this purpose, where $\tau^{\prime}$ is contained in the irrep $\Lambda$. The reason is that $K_{\Lambda, \tau^{\prime}}$ will be a function on the coset space $X^{\prime}=G / H^{\prime}$, which is different from the space $X=G / H$, where the gravitational propagators $K_{N}$ are defined. Therefore these propagators can not be multiplied and integrated to give the spin net amplitudes.

unitary irreps of the Lorentz group. At first sight this appears a natural thing to do, because the unitary irreps of the Lorentz group are also unitary irreps of the Poincare group, since the Lorentz subgroup is the little group for the zero momentum. Hence the gravitational sector would be described by the zero-momentum unitary irreps, while the matter sector would be described by the usual particle unitary irreps, having a non-zero momentum. However, because the tensor product conserves the momentum, the corresponding spin-foam amplitude would conserve the momentum of a particle propagating in a curved background.

$\S$ Except in the case of massless spin-half fermions, or if there is a Higgs scalar field. 
Note that the representation $\tau^{\prime}$ will induce a vector sub-bundle $\left(G / H, V_{\tau^{\prime}}\right)$ of the vector bundle $\left(G / H, V_{\tau}\right)$. We can then define the propagator

$$
\begin{aligned}
\tilde{K}_{\Lambda, \tau^{\prime}}(x, y) & =P_{\tau^{\prime}} U\left(x, x_{0}\right) \phi\left(g_{x}^{-1} g_{y}\right) U\left(x_{0}, y\right) P_{\tau^{\prime}} \\
& =P_{\tau^{\prime}} K_{\Lambda, \tau}(x, y) P_{\tau^{\prime}},
\end{aligned}
$$

where $P_{\tau^{\prime}}$ is a projector from $V_{\tau}$ to $V_{\tau^{\prime}}$. It is clear that $\tilde{K} \in \operatorname{Hom}\left(V^{\prime}, V^{\prime}\right)$ and it transforms correctly under $x \rightarrow x h^{\prime}$ and $y \rightarrow y \tilde{h}^{\prime}$, where $h^{\prime}, \tilde{h}^{\prime} \in H^{\prime}$.

In our case we take the index on the massless-like spin net field of spin $j$ to be from a reducible $\operatorname{Spin}(2)$ helicity representation

$$
V_{j}^{\prime}=\mathcal{L}\{|j\rangle,|-j\rangle\}
$$

This choice is motivated by the continuum space canonical analysis and with the flat space Poincare invariance. Clearly $V_{j}^{\prime}$ is a subspace of $V_{j}$, and the $\operatorname{Spin}(2)$ subgroup is generated by the $J_{z}$ component of the angular momentum.

In the $j=1 / 2$ case we have $V\left(\Lambda_{s}^{ \pm}\right)=V_{1 / 2}=V_{1 / 2}^{\prime}$, so that the only way to distinguish between the massive and a massless fermion is by a choice of the terms in the quadratic action (35).

In the $j=1$ and $S_{1}=(1 / 2,1 / 2)$ case, the index $i$ in the massless-like $A_{i}$ operator will take two values, so that the cubic interaction term would be again given by the formula (56), but now all the indices will belong to the $S O(2) V_{\frac{1}{2}}^{\prime}$ and $V_{1}^{\prime}$ representations.

Note that in the $j=1$ case one can also take $S_{1}^{+}=(1,0)$ and $S_{1}^{-}=(0,1)$ Lorentz irreps. These are the irreducible parts of the antisymmetric tensor $f_{\mu \nu}$, and we can denote the corresponding spin net fields as $f_{i}^{ \pm}(\times)$. The spin-foam analog of the action $\int d^{4} x \bar{\psi} \gamma^{\mu \nu} \psi f_{\mu \nu}$ will be given by

$$
\sum_{N} \psi_{s}^{ \pm}(\times) \psi_{s^{\prime}}^{ \pm}\left(\times^{\prime}\right) f_{i}^{ \pm}\left(\times^{\prime \prime}\right) \mathcal{V}_{ \pm \pm}^{s s^{\prime} i}\left(\times, \times^{\prime}, \times^{\prime \prime} ; \times_{1}, \ldots, \times_{5}\right) \phi_{\times_{1}} \cdots \phi_{\times_{5}}+(\text { h.c. }),
$$

and the corresponding spin net amplitude is given by

$$
A_{ \pm \pm}^{s s^{\prime} i}=\int_{X^{6}} d^{5} x d y K_{\frac{1}{2}}^{s t}\left(x_{1}, y\right) K_{1}^{i j}\left(x_{4}, y\right) K_{\frac{1}{2}}^{s^{\prime} t^{\prime}}\left(x_{2}, y\right) C_{t t^{\prime} j}^{\frac{1}{2} \frac{1}{2} 1} \prod_{k<l} K_{N_{k l}}\left(x_{k}, x_{l}\right),
$$

where $K_{1}=K_{(1,0), 1}=K_{(0,1), 1}$.

Note that the construction (59) gives for $j=2$ a massless-like helicity two field $h_{ \pm 2}(\times)$. Since this is a local excitation on a given spin net, it can 
be considered as a spin foam version of the graviton. Again there are two possible choices for the corresponding Lorentz irrep. One can take $S_{2}=(1,1)$ or $S_{2}^{+}=(2,0)$ and $S_{2}^{-}=(0,2)$. The first choice corresponds to a symmetric traceless tensor $h_{\mu \nu}$, while the second and the third choice are the irreducible pieces of the tensor $R_{\mu \nu, \rho \sigma}$, which has the symmetry properties of the Reimann curvature tensor. Clearly, we will then take the $(1,1)$ irrep for the graviton, and one would then use the $\tilde{K}_{(1,1), 2}$ propagator to construct the interaction vertices.

The realistic interactions also require an introduction of the internal symmetry group $\tilde{G}$. In the framework of the spin foam models, the simplest way to do this is to replace the category of representations $\operatorname{Cat}(G)$ with the category $\operatorname{Cat}(G \times \tilde{G})$. The matter irreps would be then given by $\left(S, \lambda_{S}\right)$, where $\lambda_{S}$ is the corresponding irrep of $\tilde{G}$. The gravitational sector irreps would be $(N, 1)$, where 1 is the trivial irrep of $\tilde{G}$. The matter action would again have the form (53) in the topological gravity case, or (54) in the non-topological gravity case, but now the products of the matter fields have to be contracted by the intertwiners $C_{\ldots \ldots}^{\lambda_{1} \cdots \lambda_{k}}$ for the internal group irreps.

\section{Conclusions}

The constructions we have presented are inspired by the formalism of particle quantum field theories, and it is a straightforward extension of the existing gravitational spin-foam models. Given that the purely gravitational models can be related to a discretized path-integral quantization of the BF theory [29], it is reasonable to expect that our model could also be related to an analogous discretized path-integral quantization of the BF theory with matter. One would then need to rewrite the Einstein-Hilbert action with matter in an appropriate form, for example

$$
\begin{aligned}
S_{4} & =\int\langle B \wedge R\rangle+\int\langle B \wedge e \wedge \bar{\psi} \gamma(\partial+\omega \gamma) \psi\rangle \\
& +\int\langle B \wedge B\rangle\left(\frac{1}{2} f^{a b} f_{a b}+g \bar{\psi} \gamma^{a} \psi A_{a}+\cdots\right),
\end{aligned}
$$

where $\omega$ is the spin connection, $R=d \omega+\langle\omega \wedge \omega\rangle$ is the corresponding curvature two-form, $A_{a}=e_{a}^{\mu} A_{\mu}, f_{a b}=e_{a}^{\mu} e_{b}^{\nu} f_{\mu \nu}$ and the dots stand for other matter fields. $\langle$,$\rangle is an appropriate contraction of the S O(3,1)$ indices $a, b, \ldots$, $\gamma$ are the appropriate gamma matrices, $\mu, \nu, \ldots$ are the curved space indices and $e_{a}^{\mu}$ are the inverse fierbeins. 
Note that we have written the matter couplings in terms of the tangent space components $A_{a}, \ldots$. These fields carry only the $S O(3,1)$ representation indices, and they would be the prime candidates for the continuous space limit of our spin-network fields. This would also mean that one needs to introduce the fierbein form $e^{a}=e_{\mu}^{a} d x^{\mu}$ in the BF formalism for matter, since it is not possible to eliminate it from the action (62) by using the constraint $B=e \wedge e$. However, in $d=3$ case there is no such problem, since $B=e$.

As far as the graviton-like field $h(\times)$ is concerned, its continuous space analog would be $h_{a b}(x)=e_{a}^{\mu} e_{b}^{\nu} h_{\mu \nu}(x)$. In order to interpret this field as the graviton, one would need to implement the constraint $h_{\mu \nu}=g_{\mu \nu}-g_{\mu \nu}^{0}$, where $g_{\mu \nu}=e_{\mu}^{a} e_{\nu a}$ is the spacetime metric, and $g_{\mu \nu}^{0}$ is a given background spacetime metric. This would then imply that the amplitude for a transition from a spatial spin net with $n$ gravitons to a spatial spin net with $m$ gravitons should not involve a sum over the background irreps $N$, since the background is fixed. That amplitude would be then given by the extension of the formula (18). Hence it is important to study further the model, in order to better understand the continuous spacetime limit, since there is a possibility that one would recover the perturbative gravity scattering amplitudes in a fixed background spacetime geometry. This also applies to the matter fields, so that this type of study will determine the correctness of our model.

The fact that we have taken the matter spin net fields in the non-topological gravity model to have indices from the representations of the $S O(3)$ and $S O(2)$ subgroups, means that the transition amplitudes will be $S O(3)$ or $S O(2)$ group covariant objects. One then wanders what happens with the Lorentz covariance. There is no such problem for the spin-half fermions, or for the $(j, 0)$ and the $(0, j)$ matter irreps. However, if we want to have the most general interactions, we need to include $(j, k)$ irreps with $j k \neq 0$. Hence we encounter a spin-foam analogue of the particle field theory spinorvector interaction problem. The only possible answer is that there should be a spin-foam version of the gauge invariance which reduces the Lorentz covariant expressions to $S O(3)$ or $S O(2)$ covariant gauge-fixed expressions. In that case the expressions (54) and (55) can be understood as gauge-fixed actions. One can think of these actions as a spin-foam generalization of the light-cone gauge field theory actions [30]. Actually, this analogy with the light-cone gauge field theory is even closer, since the finite-dimensional $S O(n)$ irreps are unitary, and hence the corresponding spin-foam model involves only the unitary representations, in contrast to the topological gravity spin foam model, where the matter irreps are pseudo-unitary. 
Obtaining the Lorentz covariant expressions in the non-topological gravity case would then amount to coupling simple unitary irreps $N$ to pseudounitary irreps $S$, and then forming the actions of the type (53). These actions should be invariant under a spin-foam version of the gauge transformations, so that one would obtain the action (54) in a particular gauge. A better understanding of these issues deserves a further study.

Note that in the case of spin foam models where $A_{2}(\Lambda)$ is not $\operatorname{dim} \Lambda$, it is not known what is the group field theory formulation. However, the field theory formulation is not necessary for the determination of the amplitudes. We used it in order to get a physical insight for our construction. Therefore, when $A_{2} \neq \operatorname{dim} \Lambda$, we will postulate the amplitude formulas (17) and (18), with $A_{e}$ and $A_{v}$ given by the same expressions as in the $A_{f}=\operatorname{dim} \Lambda$ case.

An important issue which has to be explored is the question of the perturbative finiteness of the model. As in the purely gravitational case [13, 14], this will boil down to the study of the asymptotic behaviour of the spin-net propagators $K_{N}$ and $K_{S, s}$. Note that in the Euclidian case $\operatorname{Tr} K_{S, s}$ is the same as $K_{N}$, where $N=(s, s)$, so that one expects that the Euclidian spinfoam matter model should be finite. In the Lorentzian case there is no such a simple relation between the propagators, so that one needs to make a more detailed analysis. Alternatively, one can formulate a quantum group version of the construction we gave, which could be made automatically finite if the gravitational irreps are from a finite set, and if there are finitely many matter irreps.

The issue of the semiclassical/continuous space limit of the spin foam models needs a more detailed study. There are no rigorous statements about the semiclassical theory. In the purely gravitational case one can show that in the limit of large angular momenta one obtains amplitudes which are sums of terms proportional to $\exp \left( \pm i S_{R}\right)$, where $S_{R}$ is the Regge form of the EinsteinHilbert action [31]. Actually, because of the form of the propagator $K_{N}$, see (30) and (47), this seems to be a good approximation even for arbitrary values of the angular momenta. Hence there are good indications that one is on the right track. In the case of matter, one would then need to obtain expressions containing $\exp \left( \pm i S_{R} \pm i S_{s m}\right)$, where $S_{s m}$ is the simplical version of the matter actions [32]. This type of analysis could also settle the question of which fields are really massive and which ones are massless.

\section{Acknowledgements}

I would like to thank L. Crane and R. Picken for the discussions. 


\section{References}

[1] J.W. Barrett and L. Crane, J. Math. Phys. 39 (1998) 3296

[2] J.W. Barrett and L. Crane, Class. Quant. Grav. 17 (2000) 3101

[3] A. Perez and C. Rovelli, Nucl. Phys. B 599 (2001) 255

[4] A. Perez and C. Rovelli, Phys. Rev. D 63 (2001) 041501

[5] A. Miković, Class. Quant. Grav. 18 (2001) 2827

[6] V.G. Turaeev and O.Y. Viro, Topology 31 (1992) 865

[7] L. Crane, D.H. Kauffman and D.N. Yetter, J. Knot Th. Ramif. 6 (1997) 177, hep-th/9409167

[8] D.V. Boulatov, Mod. Phys. Lett. A7 (1992) 1629

[9] H. Ooguri, Mod. Phys. Lett. A7 (1992) 2799

[10] R. De Pietri, L. Freidel, K. Krasnov and C. Rovelli, Nucl. Phys. B 574 (2000) 785

[11] M. Reisenberger and C. Rovelli, Class. Quant. Grav. 18 (2001) 121

[12] R. Levine, A. Perez, C. Rovelli, gr-qc/0102051

[13] A. Perez, Nucl. Phys. B 599 (2001) 427

[14] L. Crane, A. Perez and C. Rovelli, Phys. Rev. Lett. 87 (2001) 181301

[15] L. Crane, gr-qc/0004043

[16] L. Crane, gr-qc/0110060

[17] S. Weinberg, The Quantum Theory of Fields, vol. 1, Cambridge University Press (1995) Cambridge

[18] C. Rovelli and L. Smollin, Phys. Rev. D 52 (1995) 5743

[19] R. Oeckl and H. Pfeiffer, Nucl. Phys. B 598 (2001) 400

[20] H. Pfeiffer, hep-th/0106029 
[21] H.A. Morales-Tecotl and C. Rovelli, Phys. Rev. Lett. 72 (1994) 3642

[22] J. Baez and K. Krasnov, J. Math. Phys. 39 (1998) 1251

[23] J. Baez, Lect. Notes Phys. 543 (2000) 25

[24] D. Oriti, Rept. Prog. Phys. 64 (2001) 1489

[25] J.W. Barrett, Adv. Theor. Math. Phys. 2 (1998) 593

[26] L. Freidel and K. Krasnov, J. Math. Phys. 41 (2000) 1681

[27] R. Camporesi and A. Higuchi, J. Geom. Phys. 15 (1996) 1, grqc/9505009

[28] W. Ruhl, The Lorentz Group and Harmonic Analysis, WA Benjamin Inc (1970) New York

[29] L. Freidel and K. Krasnov, Adv. Theor. Math. Phys. 2 (1999) 1183

[30] W. Siegel, Introduction to String Field theory, World Scientific (1988) Singapoore, hep-th/0107094

[31] J.W. Barrett and R.M. Williams, Adv. Theor. Math. Phys. 3 (1999) 1

[32] H-C. Ren, Nucl. Phys. B 301 (1988) 661 


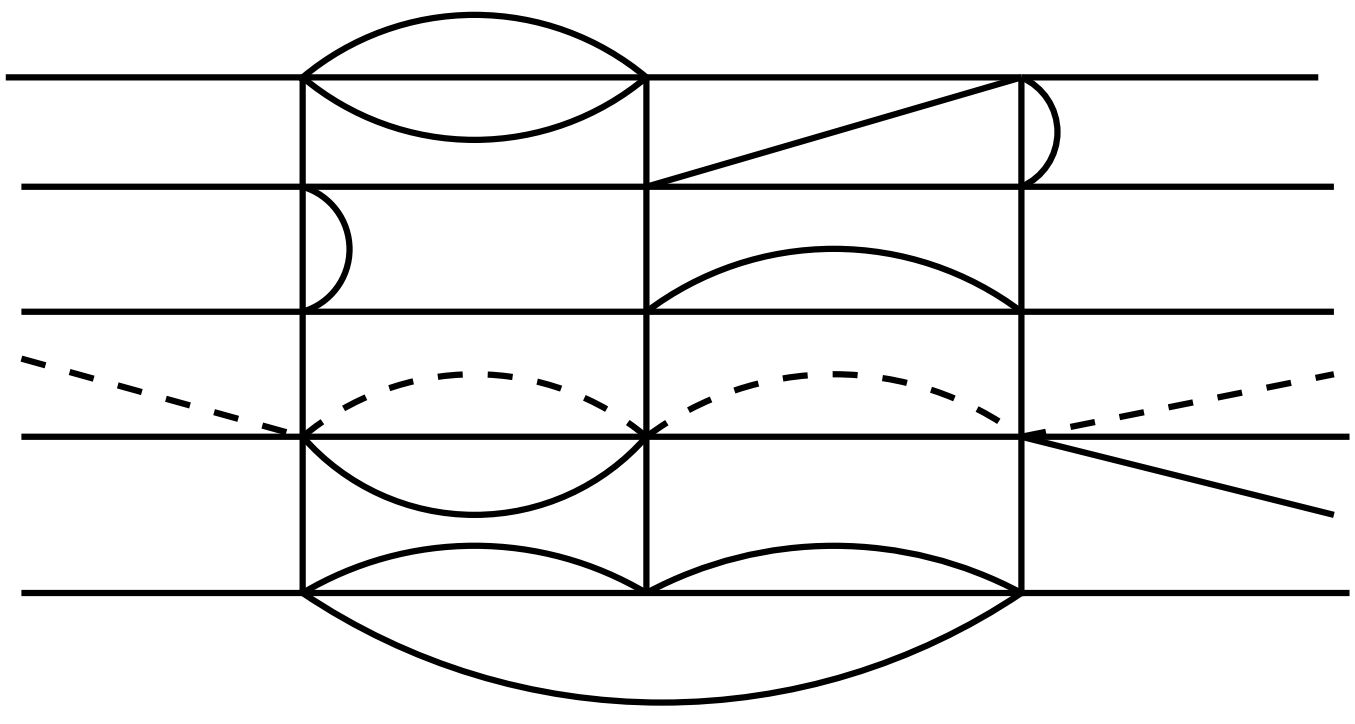

Figure I: A spin foam Feynman diagram describing propagation of a matter field, which is represented by a dotted line. 


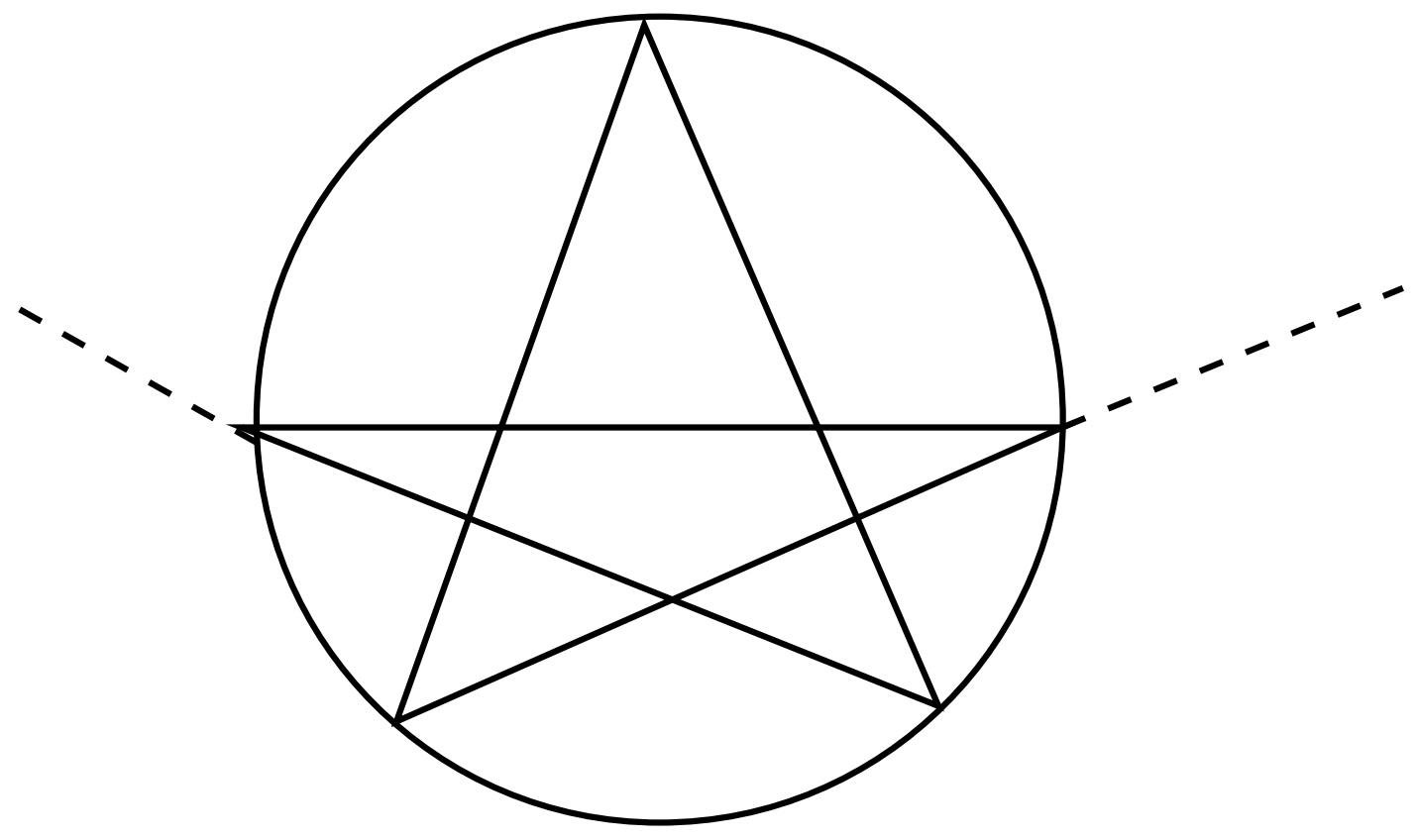

Figure II: The simplest spin net graph which gives a 7 -valent vertex for a spin foam Feynman diagram.

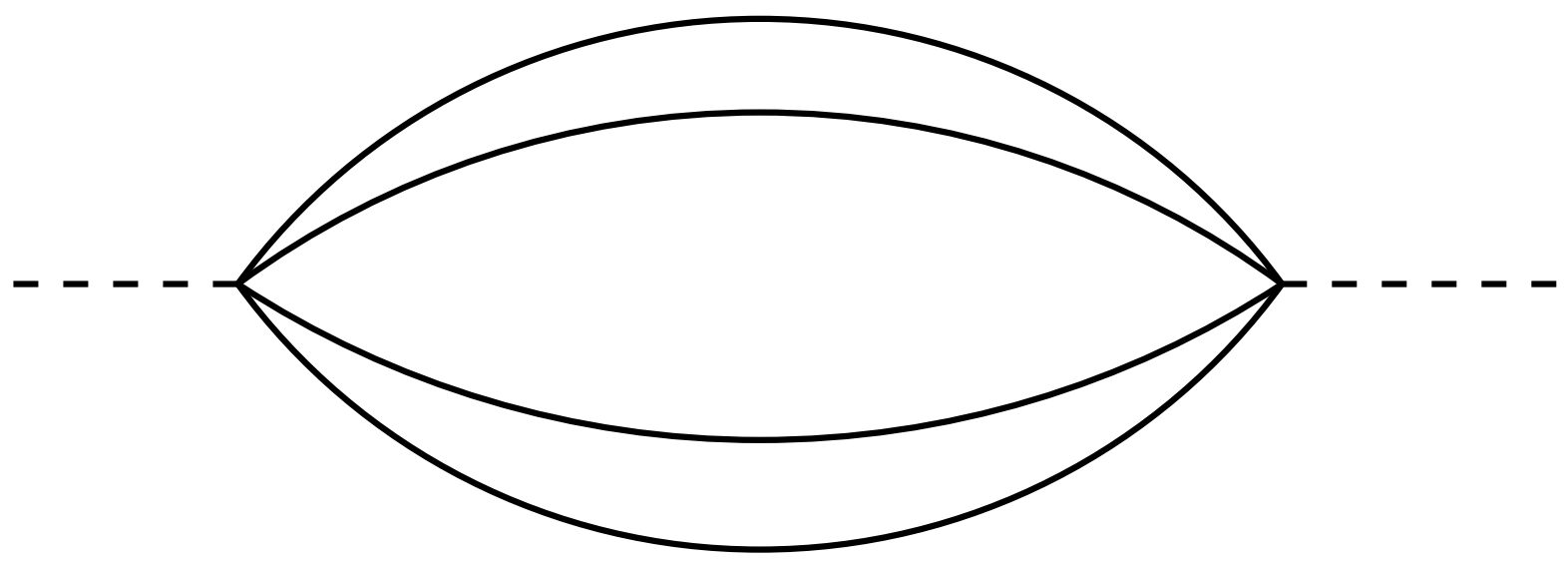

Figure III: A nontrivial spin net graph which gives a matter propagator for a spin foam Feynman diagram. 


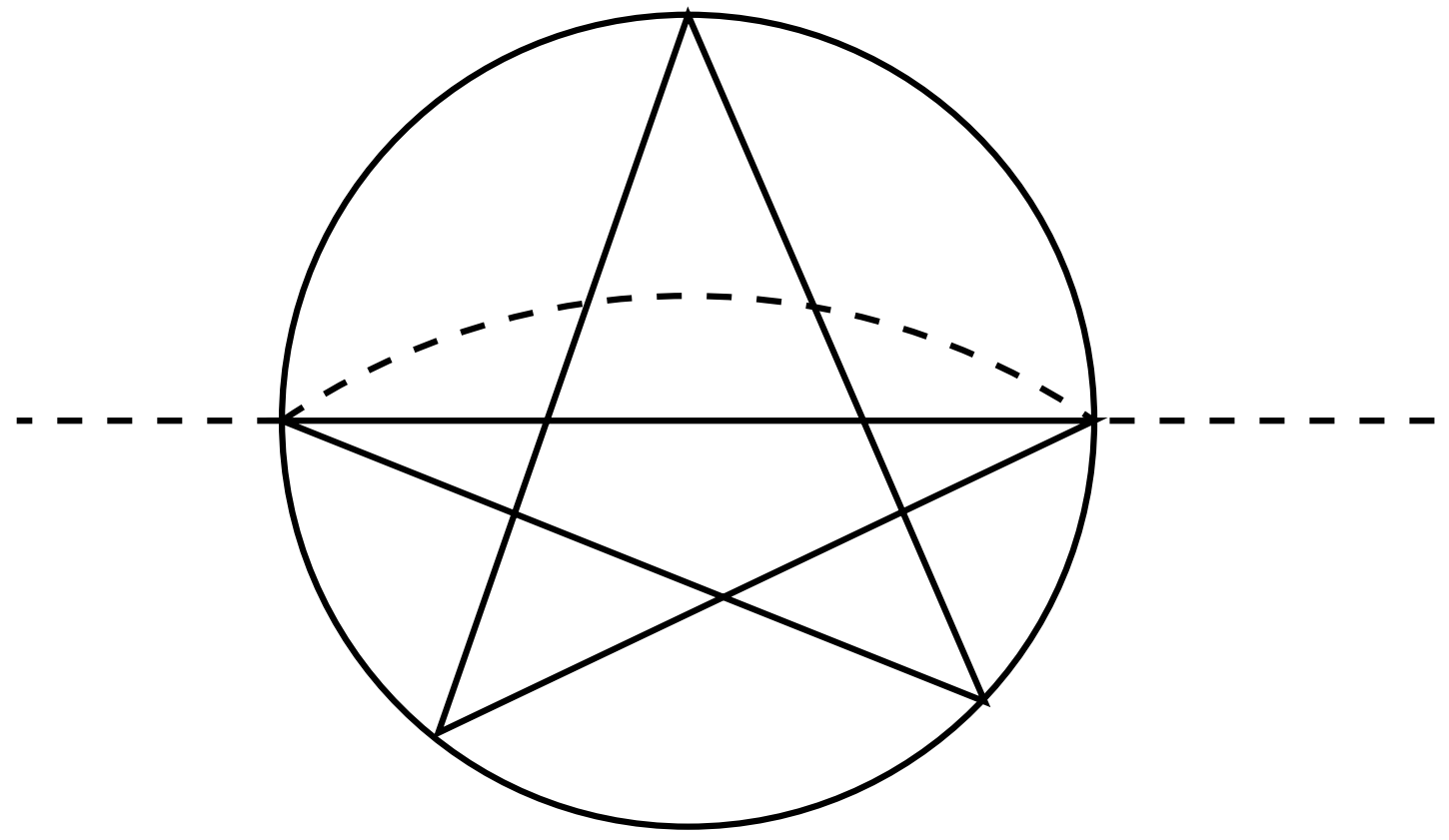

Figure IV: An example of a more complex spin net graph determining the 7 -valent spin foam vertex.

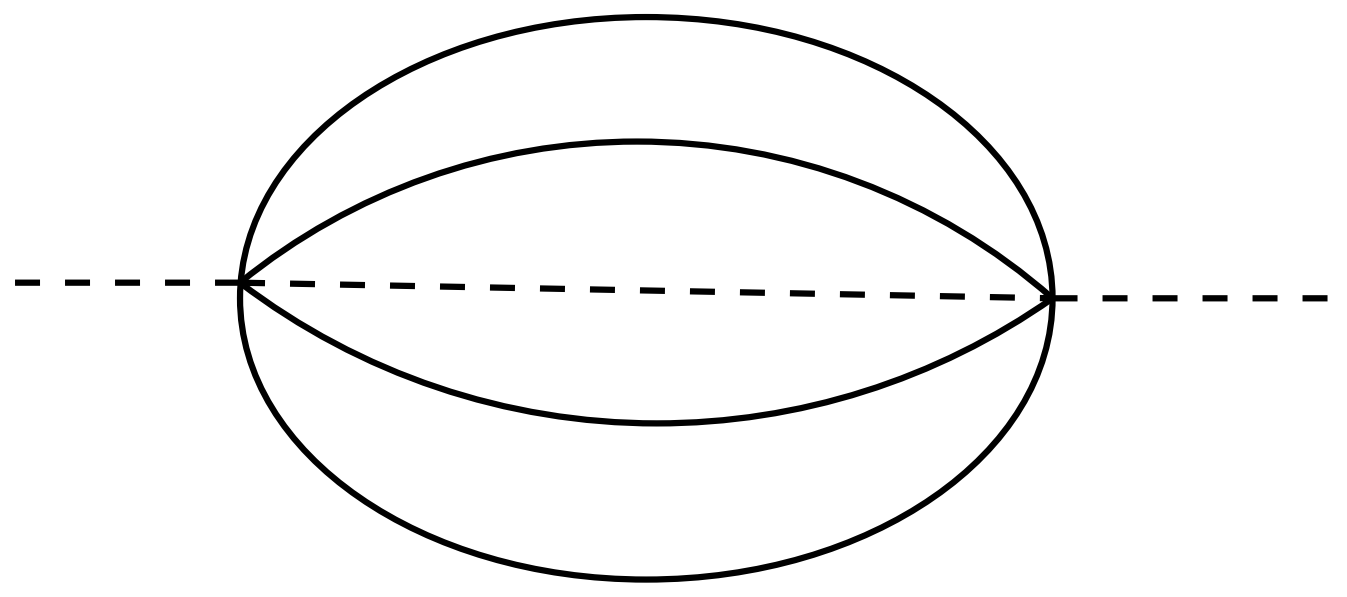

Figure V: A more complex spin net graph determining the matter spin foam propagator. 

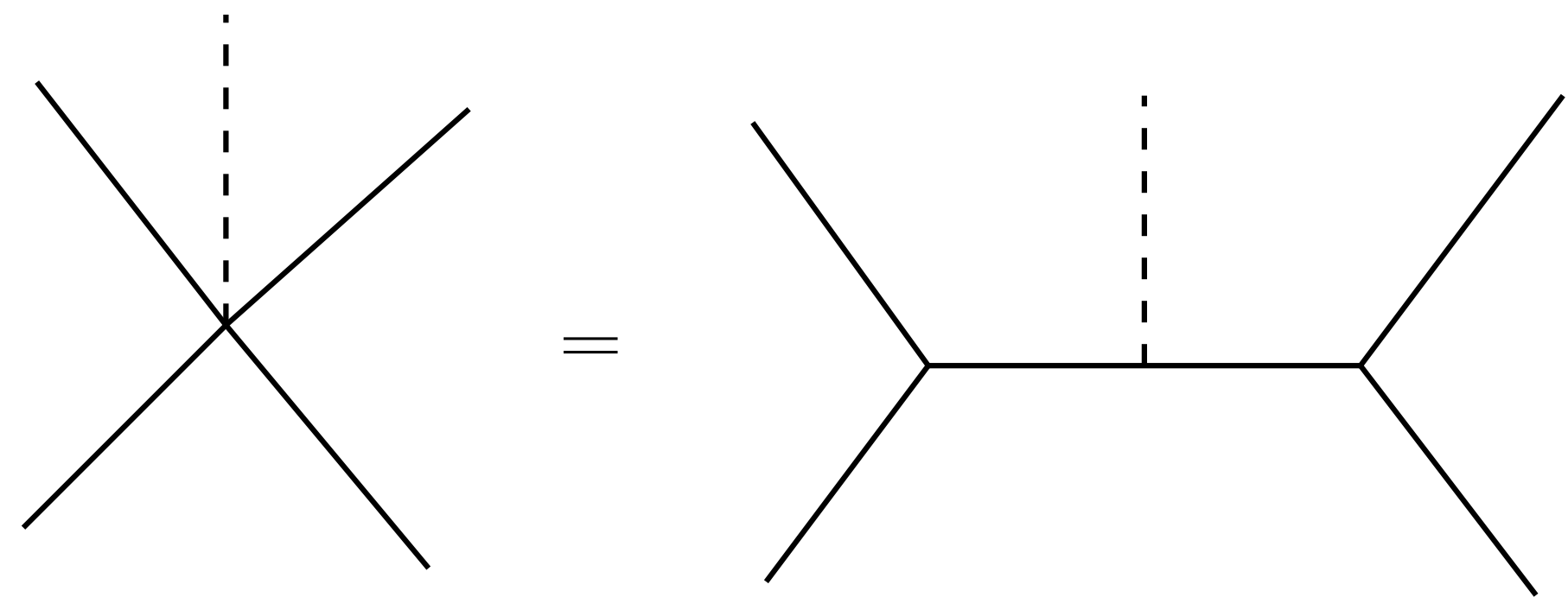

Figure VI: Diagrammatic representation of the equation (23).
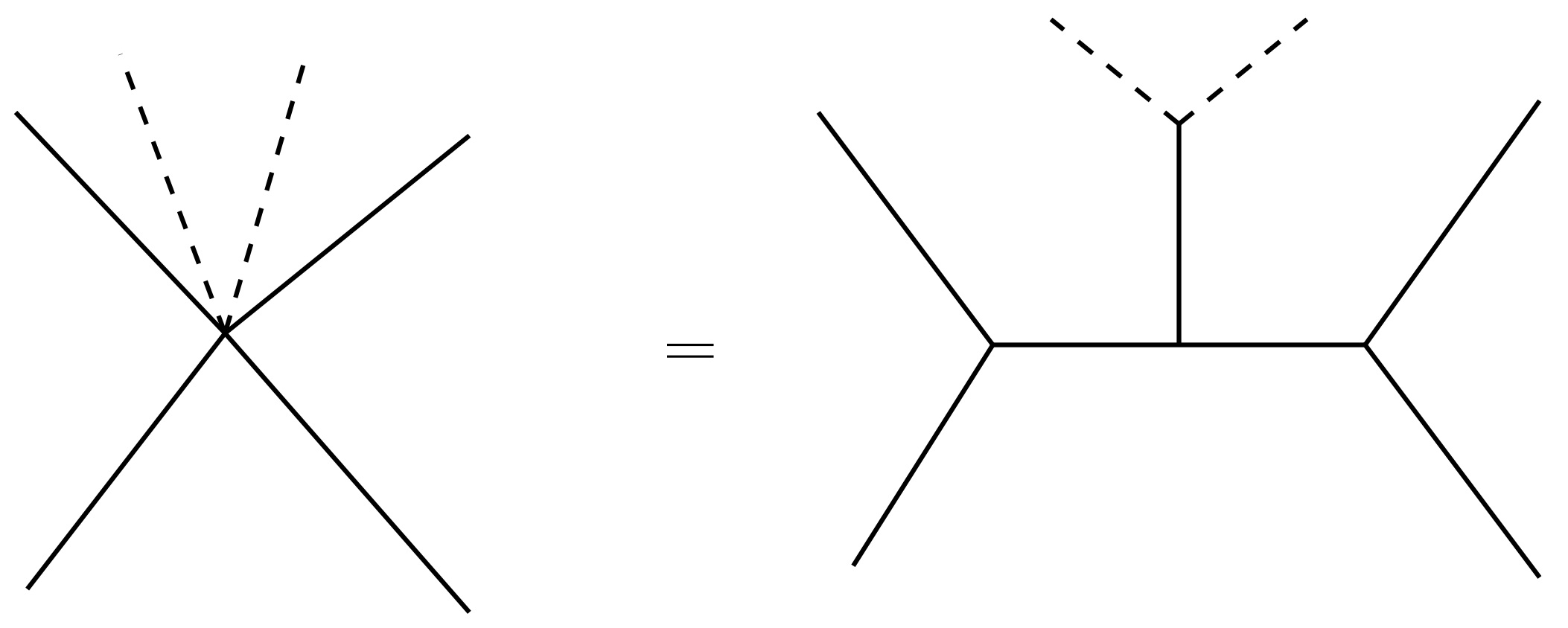

Figure VII: Diagrammatic representation of the equation (25). 


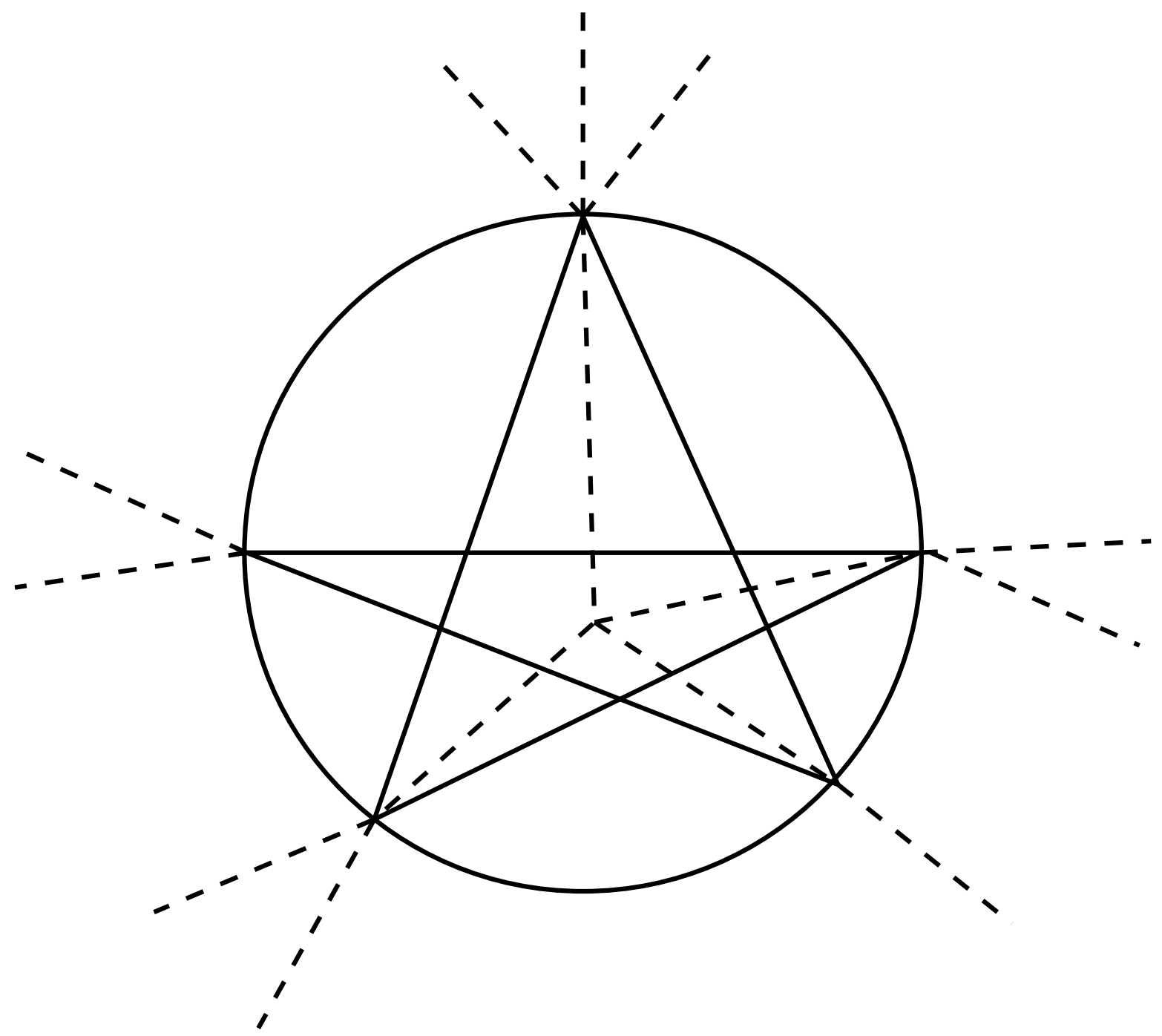

Figure VIII: A spin net graph determining a 15-valent spin foam vertex. 


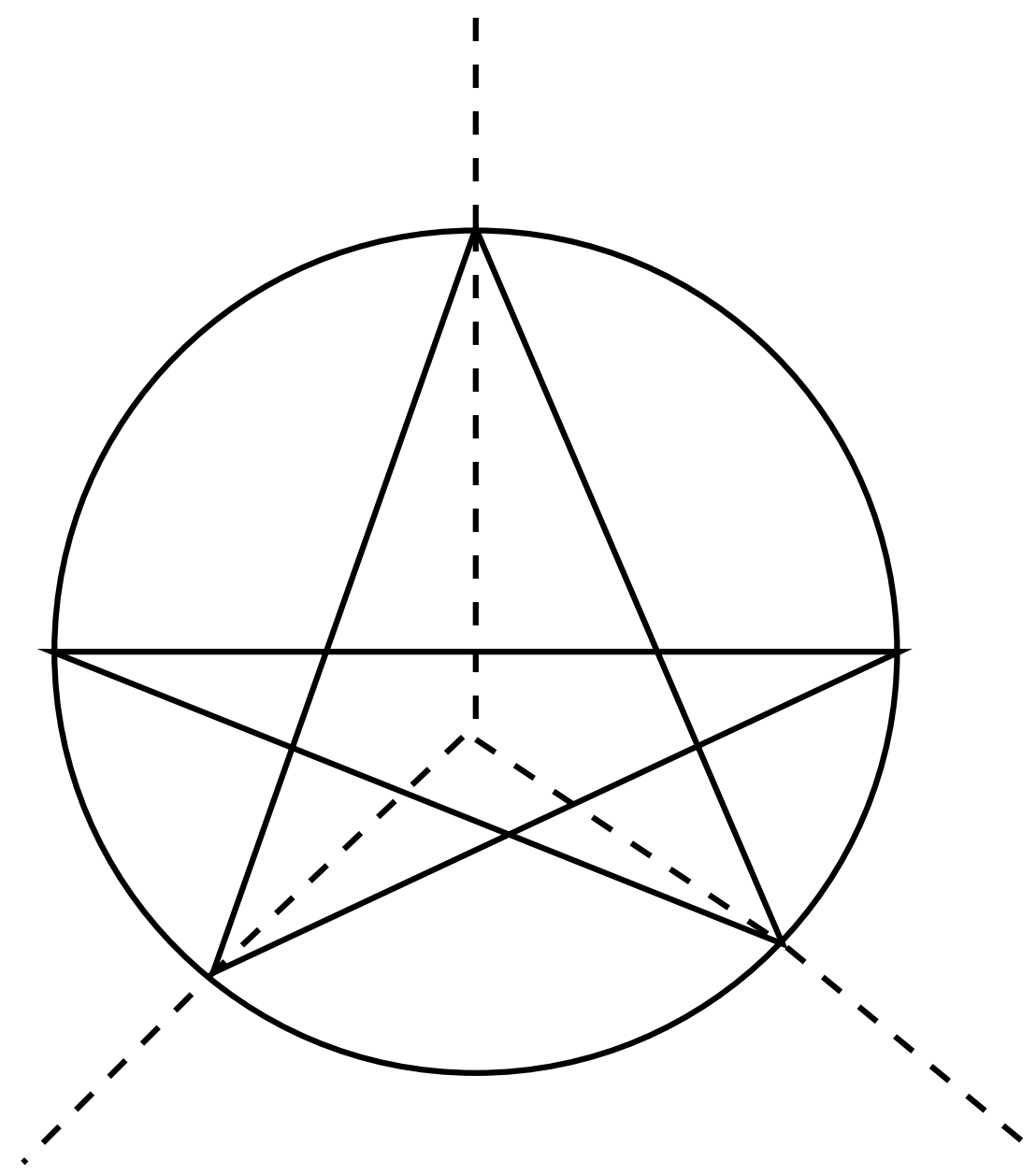

Figure IX: The spin net graph for the spin foam vector-spinor interaction vertex. 